\title{
COVID-19: ALTERNATIVAS Y SOLUCIONES. UNA MIRADA DESDE LA INVESTIGACIÓN UNIVERSITARIA.
}

Desde finales del pasado año 2019 y durante todo este año 2020 los ojos del mundo han estado enfocados en un propósito común: combatir el coronavirus que causa la afectación de la COVID-19. No sólo desde el punto de vista médico con el fin de encontrar una vacuna, sino en todas las áreas del accionar humano a propósito de mitigar los estragos que se han derivado de esta nueva pandemia mundial que ya se ha cobrado la vida de casi cien mil personas alrededor del mundo.

A razón de esto, SUMMA. Revista disciplinaria en ciencias económicas y sociales, unidad estratégica adscrita a la Coordinación de investigación de la Corporación Universitaria AUTÓNOMA DE NARIÑO - extensión Santiago de Cali, en apropiación de su compromiso social y académico, brinda su espacio de divulgación y socialización para que docentes e investigadores nacionales y extranjeros muestren a la comunidad científica sus aportaciones de alternativas y soluciones ante esta crisis sanitaria. Confiando inequívocamente en las fortalezas de las instituciones de educación superior en sus funciones de docencia, investigación y extensión auguramos que esta mirada universitaria frente a la COVID-19 genere aportes significativos de cara a una salida necesaria de esta pandemia mundial. Así, con el mayor beneplácito entrego en manos de nuestros respetados aliados esta edición Año 2 Número Especial 2020 donde hemos contado con la impecable participación de autores procedentes de Colombia, Ecuador y Venezuela.

La entrega inicia con un cuerpo de notas científicas donde Betty De La Hoz Suárez y Nelly Panchi Castro presentan el trabajo Asistencia social a migrantes venezolanos durante la pandemia de la COVID19 en Ecuador. Luego se puede conocer sobre COVID-19 y empresas de plataforma tecnológica: sector del mercado bursátil más atractivo para invertir, de los autores Diego Díaz Castro, Rodolfo Gómez Torres y Víctor Salazar Martínez. Después, Juan Quintero Rivera muestra sus conclusiones sobre el Producto interno bruto de Colombia y alternativas económicas frente al COVID-19. Desde un 
enfoque educativo, Nataliya Barbera Alvarado, Efraín Hernández Buelvas y Arney Vega Martínez exponen sobre los Desafíos de la gestión pedagógica en la virtualidad ante la crisis del COVID-19. Por su parte, Jacqueline Garcés Fuenmayor y Carlos Mora Bolaños esbozan acera de las Estrategias de aprendizaje para mitigar la deserción estudiantil en el marco de la COVID-19.

Seguidamente, la sección de artículos inicia con el trabajo de María Useche Aguirre, Flor Salazar Vázquez, Christian Barragán Ramírez y Paola Sánchez Salazar titulado Horizontes estratégicos empresariales en América latina ante la pandemia generada por la COVID-19. Luego, en la investigación de Juan Araujo Cuauro se puede conocer sobre la Violación de los derechos humanos en Venezuela: la otra cara de la pandemia; bajo ese mismo contexto geográfico, Milagros Rodríguez e Isabel Peleteiro Vázquez exponen su estudio sobre Antropolítica en Venezuela: un cuenco de mendigo, más aún en tiempos de pandemia 2020. Desde una perspectiva empresarial, Aminta De La Hoz, Martha González Vergara y Emily Lugo Hernández presentan la Aplicabilidad del principio contable de negocio en marcha en tiempos de COVID-19.

Los investigadores Álvaro Álvarez Rojas, Magle Sánchez Castellanos y Carlos Rojas Fernández comparten su propuesta de un Objeto virtual de aprendizaje para el diseño de prototipos de robótica: estrategia didáctica ante el COVID-19. Por su parte Ernesto García Cali, Ana Valle Ospino y José Cepsedes Garrido presentan un trabajo sobre Employer branding. Reflexiones en tiempos de pandemia. En esta misma línea, Jacob Rivera Hernández, María Moreno Hernández, Sonia Ethel Durán y Jesús García Gulliany exponen sobre la Educación en tecnología como paradigma didáctico, innovador e inclusivo en el marco del COVID-19. Finalmente, se encuentra el trabajo de Carlos Arana Castañeda titulado "El oriente de Cali no tiene ni dios ni ley": redes sociales digitales y violencia racializada a propósito de la COVID-19.

Una vez más, gracias a todos los articulistas de esta edición por confiar en SUMMA. Revista disciplinaria en ciencias económicas y 
sociales como la ventana para la difusión de sus resultados de investigación. Muchas gracias también a todos los pares evaluadores externos que contribuyeron en garantizar la calidad y rigurosidad científica de nuestra publicación. Y gracias a ustedes, apreciados lectores, por acompañarnos en cada entrega. Nos complace informarles que a partir de este número nuestra revista cuenta con la asignación de identificadores de objetos digitales (DOI, por sus siglas en inglés) como un peldaño más que nos apalanca en nuestra política de calidad editorial.

MSc. Josnel Martínez Garcés

Editor en Jefe 